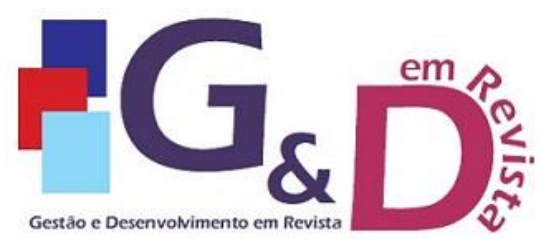

Gestão e Desenvolvimento em Revista V. 8, N. 2, jul-dez/2021, p. 90-108.

ISSN online: $2446-8738$

Artigo recebido em: 19/04/2021

Artigo aprovado em: 25/01/2022

\title{
ANÁLISE ECONOMÉTRICA-ESPACIAL DO IDHM DO ESTADO DO MARANHÃO
}

\author{
Marieli Vieira \\ Doutoranda em Economia do Desenvolvimento pela Universidade Federal do Rio Grande do Sul - \\ UFRGS \\ E-mail: marihvieira18@gmail.com
}

\author{
Caroline Todeschini \\ Doutoranda em Economia do Desenvolvimento pela Universidade Federal do Rio Grande do Sul - \\ UFRGS. \\ E-mail: carolinetdsc@gmail.com \\ José Luiz Parré \\ Doutor em Economia Aplicada pela Escola Superior de Agricultura Luiz de Queiroz. Atualmente é \\ Professor Titular do Departamento de Economia da Universidade Estadual de Maringá - UEM. \\ E-mail: jlparre@uem.br
}

\author{
Bruno Santos Baggi \\ MBA em Análise Econômica pela Fundação Instituto de Pesquisas Econômicas - FIPE/USP. \\ Atualmente é Analista Financeiro no Universo Online S.A. UOL. \\ E-mail: brunobaggi@gmail.com
}

\begin{abstract}
Resumo
O presente trabalho buscou analisar a dependência espacial do desenvolvimento econômico em termos de Índice de Desenvolvimento Humano Municipal (IDHM) nos municípios do estado do Maranhão no ano de 2010 e algumas variáveis que podem afetá-lo. Foram empregados técnicas de análise exploratória de dados espaciais (AEDE). Foi calculada a estatística I de Moran para verificar a existência de autocorrelação espacial, sendo que essa indicou a formação de clusters significativos em todas as regiões do estado. O Modelo de Erro Espacial (SEM) foi utilizado para regredir a proporção de domicílios com banheiro e idade do município, que mostraram relação positiva; o percentual de população até 10 anos de idade e PEA da agropecuária, com relação negativa; e o IDHM dos municípios com o número de médicos para cada mil habitantes e fertilidade das mulheres até 17 anos de idade, que não foram estatisticamente significativos. A taxa de atividade de crianças entre 10 e 14 anos, ao contrário do esperado, afetou positivamente o IDHM dos municípios maranhenses, indicando que a contribuição dessa força de trabalho para a geração de renda pode ser maior que seu impacto negativo na educação. Além disso, verificou-se dependência espacial dessas variáveis, indicando que elas afetam também os municípios vizinhos.
\end{abstract}

Palavras-chave: AEDE. Economia regional. Desenvolvimento econômico.

\begin{abstract}
This study aimed to analyze the spatial dependence of economic development in terms of Municipal Human Development Index (IDHM) in the municipalities of the Maranhão state in 2010 and some variables that can affect it. Exploratory spatial data analysis (AEDE) techniques were used. The Spatial Error Model (SEM) was used to regress the proportion of households with bathroom and age of the municipality, which showed a positive relationship; the percentage of population up to 10 years of age and PEA in agriculture, with negative relationship; and the IDHM of the municipalities with the number of doctors per thousand inhabitants and fertility of women up to 17 years of age, who were not statistically significant. The children's activity rate between 10 and 14 years old, contrary to expectations, it positively affected the IDHM of Maranhão municipalities, indicating that the contribution of this workforce to income generation may be greater than its negative impact on education. Furthermore, there was spatial dependence of these variables, indicating that they also affect neighboring municipalities.
\end{abstract}

Key words: AEDE. Regional economy. Economic development. 


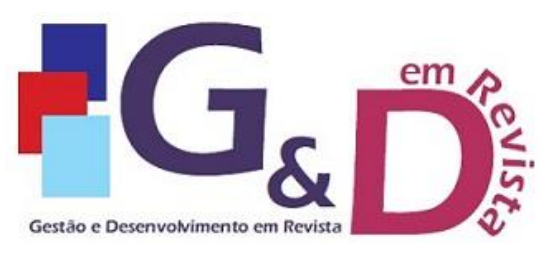

Gestão e Desenvolvimento em Revista V. 8, N. 2, jul-dez/2021, p. 90-108.

ISSN online: $2446-8738$

Artigo recebido em: 19/04/2021

Artigo aprovado em: 25/01/2022

\section{INTRODUÇÃO}

O Brasil pode ser considerado um país heterogêneo quando se considera o nível de desenvolvimento econômico. Tanto o tamanho do seu território quanto a própria dinâmica do processo de desenvolvimento contribuem para essa realidade. As facilidades geográficas e incentivos fiscais contribuem para a concentração das atividades econômicas em determinadas regiões, promovendo 0 seu desenvolvimento por meio da dinâmica de crescimento que desencadeia, enquanto outras regiões permanecem estagnadas.

Em duas décadas, o Índice de Desenvolvimento Humano Municipal (IDHM) do Brasil saiu de muito baixo desenvolvimento humano, 0,493 em 1991, para a posição de médio desenvolvimento em $2000(0,612)$ e chegou ao nível de alto desenvolvimento em 2010 (0,727). Dados da PNAD (Pesquisa Nacional por Amostra de Domicílios) indicaram que, em 2016, o IDHM brasileiro havia aumentado para 0,776, mantendo-se no nível de alto desenvolvimento humano (ATLAS BRASIL, 2020).

Porém, muitas regiões ainda permanecem aquém do desenvolvimento desejado. Em 1991, o estado com o IDHM mais baixo era o Maranhão $(0,357)$. Quase vinte anos depois, em 2010, esse estado detinha o segundo IDHM mais baixo $(0,639)$, ficando à frente apenas do estado de Alagoas. Em 2016, o estado do Maranhão retornou à última colocação em termos de IDHM $(0,682)$. Juntamente com Alagoas, Piauí e Pará, o Maranhão compõe o grupo de estados com desenvolvimento considerado médio, enquanto o restante do país apresenta indicadores de alto desenvolvimento. A discrepância regional e o fato deste estado estar aquém da média nacional justificam a tentativa de aprofundamento sobre a interação espacial entre os municípios.

Lins et al. (2014) demonstrou, por meio de econometria espacial, fortes indícios de dependência espacial positiva no IDHM dos municípios da região nordeste brasileira entre os anos de 2000 e 2010. Ou seja, os autores constataram que o nível de desenvolvimento de um município pode afetar o desenvolvimento de seus vizinhos. Lobo (2017) busca identificar padrões espaciais do IDHM educacional e verificar sua relação com o gasto médio por aluno, contanto com o auxílio da AEDE. 
Também através de análise espacial, Santos (2018) constatou que no estado do Maranhão questões de saúde municipal podem ser afetadas por municípios vizinhos. Com base em dados de óbitos ocorridos por tuberculose entre os anos 2010 e 2015, o autor constatou dependência espacial entre as taxas de mortalidade.

Tendo em vista o exposto, o objetivo deste trabalho é realizar a análise espacial do desenvolvimento econômico do estado do Maranhão por meio de procedimento econométrico visando explicar como algumas variáveis de caráter multidimensional podem influenciar o desenvolvimento econômico de um município e de seus vizinhos. A hipótese norteadora da pesquisa é de que existem padrões espaciais entre o IDHM dos municípios e as variáveis consideradas, acrescentando à literatura um estudo que busca verificar se os determinantes da qualidade de vida no estado vão além da renda.

O IDHM do ano de 2010 foi utilizado como proxy de desenvolvimento econômico e a justificativa se dá pelo fato deste ser calculado com base nas informações do Censo Demográfico, cuja última aplicação foi no respectivo ano. A fim de captar a dependência espacial e o caráter multidimensional do índice, foram consideradas variáveis que possuem relação com as áreas de educação, saúde e renda, mas que não compõem a metodologia do IDHM. Para análise espacial, foi utilizada a estatística de I de Moran Local, Diagrama de dispersão de Moran, Mapa LISA e modelo de erros espaciais.

Além desta introdução, a segunda seção deste trabalho possui uma revisão teórica sobre desenvolvimento econômico. Na terceira seção, por sua vez, apresenta-se a metodologia e o tratamento dado às variáveis nesta pesquisa. A seção quatro tece os resultados e discussões e, finalmente, as considerações finais na última seção.

\section{DESENVOLVIMENTO ECONÔMICO}

Considerando que crescimento econômico difere de desenvolvimento econômico, pode-se dizer que enquanto o primeiro abrange apenas o aumento da renda per capita, o segundo considera melhorias de indicadores econômicos, sociais 
e ambientais na medida em que a economia em questão passa de arcaica para moderna e ocorre aumento do bem estar da população (SOUZA, 2007).

Nesse contexto, o sentido de desenvolvimento econômico funde-se com 0 de desenvolvimento humano que, segundo Sen (2000), é frequentemente entendido como o processo de expansão da educação, dos serviços de saúde e de outras condições da vida humana. Ademais, as oportunidades sociais de educação e saúde complementam oportunidades individuais de participação política e econômica.

Segundo o Programa das Nações Unidas para o Desenvolvimento (PNUD):

\begin{abstract}
Diferentemente da perspectiva do crescimento econômico, que vê o bemestar de uma sociedade apenas pelos recursos ou pela renda que ela pode gerar, a abordagem de desenvolvimento humano procura olhar diretamente para as pessoas, suas oportunidades e capacidades. A renda é importante, mas como um dos meios do desenvolvimento e não como seu fim. É uma mudança de perspectiva: com o desenvolvimento humano, o foco é transferido do crescimento econômico, ou da renda, para o ser humano (PNUD BRASIL, 2021).
\end{abstract}

Existem diversas teorias que estudam o processo de desenvolvimento econômico. Em grande parte delas, constata-se que nas fases iniciais do desenvolvimento a população total concentra-se majoritariamente no meio rural e que a maior parte da riqueza gerada provém do setor primário (SOUZA, 2007).

Para ser menos dependente e estar menos vulnerável a crises internacionais, um país precisa ter uma balança comercial diversificada e maior dinamismo interno. A passagem de uma economia arcaica para uma moderna ocorre por meio da industrialização e aumento da produtividade do trabalho. Ao passo que ocorre maior oferta de empregos no meio urbano e a agricultura se torna mecanizada, a mão de obra migra do campo para a cidade. Ademais, é importante salientar que o capital investido no setor industrial e tecnológico provém dos lucros no campo.

Souza (2007) destaca ainda a importância de se considerar outros indicadores que refletem melhorias sociais e econômicas, como alimentação, atendimento médico e odontológico, segurança, qualidade do meio ambiente, mortalidade infantil e distribuição de renda.

Nesse sentido, percebe-se a importância de considerar a realidade da região no qual o objeto de estudo está localizado, uma vez que podem ocorrer permutas constantes de informações, bens, serviços, mão de obra e transbordamento de políticas públicas. 
Todavia, o processo de desenvolvimento econômico não ocorre do mesmo modo em todas as regiões. Ele se apresenta de forma heterogênea uma vez que cada região apresenta particularidades que interferem no seu processo.

No que tange as teorias de desenvolvimento com ênfase em fatores de aglomeração, François Perroux afirma que o crescimento se apresenta em polos e uma indústria, além de contribuir para o crescimento global do produto, promove no ambiente no qual está inserida um crescimento devido às indústrias motrizes e ao desencadeamento de novas necessidades coletivas (CAVALCANTE, 2008).

Já Myrdal elabora o conceito de causação circular e acumulativa da renda como uma explicação para a heterogeneidade do crescimento, uma vez que as forças do mercado operariam na geração de desigualdades. Hirschman, por sua vez, se atém no conceito de "efeitos para trás e para frente" para expressar as externalidades de um empreendimento na região na qual ele está inserido (CAVALCANTE, 2008).

Nota-se, portanto, que diversos fatores podem afetar o desenvolvimento econômico de uma localidade, podendo ser de ordem econômica, social, ambiental e política, ou ainda, geográfica, histórica e cultural.

\section{METODOLOGIA}

Nesta seção serão abordados os aspectos referentes aos métodos adotados para atingir os objetivos deste trabalho, assim como as caraterísticas das variáveis utilizadas.

\subsection{ESTATÍSTICA I DE MORAN}

Uma das estatísticas mais utilizadas para testar a dependência espacial é a estatística I de Moran, que pode ser aplicada diretamente à variável $y_{i}$, ou aos conjuntos da regressão de $y_{i}$ contra algumas variáveis explicativas.

Conforme especificam Cliff e Ord (1981), a estatística I de Moran Global pode ser expressa como:

$$
I_{t}=\left(\frac{n}{S_{0}}\right)\left(\frac{z_{t}^{\prime} W z_{t}}{z_{t} z_{t}}\right) \quad \mathrm{t}=1, \ldots, \mathrm{n}
$$


Onde $z$ é o vetor de $n$ observações para o ano $t$ na forma de desvio em relação à média. $W$ é a matriz de pesos espaciais, sua diagonal de elementos $W_{i i}$ é igual a zero e os elementos $W_{i j}$ indicam a forma como um município está conectado com outro. O termo $S_{o}$ é um escalar igual a soma de todos os elementos da matriz $W$.

O Índice de Moran Local, por sua vez, proposto por Anselin (1995), testa a autocorrelação local a fim de identificar objetos espaciais com influência no indicador Moran Global. Essa metodologia considera a análise das covariâncias entre as diferentes unidades de área.

Portanto, enquanto o Índice Global de Moran avalia a interdependência espacial entre todos os polígonos em análise, o Índice Local de Moran analisa a covariância entre um determinado polígono e certa vizinhança definida em função de uma distância $d$. Anselin (1995) define o Índice Local de Moran como produto do resíduo no polígono de referência com a média local dos resíduos dos seus vizinhos adjacentes.

Assim:

$$
I_{l}=z_{i} \sum_{j} w_{i j} z_{j}
$$

Onde $W_{i j}$ representa o valor na matriz de vizinhança para a região $i$ com a região jem função da distância $d$, e $z_{i}$ e $z_{j}$ são os desvios em relação à média.

A matriz $W_{i j}$ define os vizinhos de certo polígono. Este trabalho utiliza o critério de contiguidade, na qual duas regiões são consideradas vizinhas se elas fizerem fronteira física, pois dessa forma considera-se que seja maior a interação espacial. Quanto às fronteiras físicas em comum, conforme explica Almeida (2004), é possível levar em conta as fronteiras que tenham extensão diferente de zero e também aquelas que tenham apenas vértices em comum. Considerando essas proximidades, as matrizes de contiguidade podem ser do tipo 'rainha', 'torre' ou 'bispo'. A primeira leva em conta fronteiras de extensão diferentes de zero e os vértices, a segunda, apenas as fronteiras, enquanto a terceira leva em conta apenas os vértices. A escolha da matriz de peso espacial será entre o tipo 'rainha' ou 'torre', por serem as opções que consideram fronteiras físicas, e se dará em função do cálculo do I de Moran, tendo como objeto a regressão por Mínimos Quadrados Ordinários. 
Quando a estatística I de Moran Local fica mais próxima de +1 , isso indica que existe autocorrelação espacial positiva, ou seja, os valores altos de IDHM tendem a estar localizados perto de vizinhos que também tenham valores altos no IDHM, assim como valores baixos de IDHM tendem a estar localizados perto de vizinhos com valores baixos no IDHM. Quando o I de Moran Local for próximo de -1 isso indica que existe autocorrelação negativa, o que significa que vizinhos com valores altos de IDHM estarão próximos de vizinhos com baixos valores de IDHM e vice-versa.

Após a estimativa do I de Moran Local, faz-se interessante gerar um mapa indicando quais regiões apresentam correlação local. Esse mapa é chamado por Anselin (1995) de LISA (Local Indicators of Spatial Association) e é indicado para verificar a autocorrelação espacial local juntamente com as características do espaço, ou seja, visa explanar áreas com dinâmica espacial própria que merecem análise.

O diagrama de dispersão de Moran é outra forma de interpretar a estatística I de Moran. Por meio do diagrama pode-se visualizar a correlação linear de uma variável para cada uma das unidades (o IDHM nesse caso) nas abscissas e, nas ordenadas, a média do valor padronizado da variável para os municípios vizinhos. Desta forma, como o diagrama de Moran é dividido em quatro quadrantes, os valores que estiverem acima da média relacionados com vizinhos que estiverem também acima da média ficarão no primeiro quadrante. $O$ segundo e o quarto quadrante mostram os valores altos cercados de valores baixos e os valores baixos cercados de valores altos, respectivamente. $\mathrm{E}$ os valores abaixo da média cercados por vizinhos que também tenham valores abaixo são representados no terceiro quadrante (MONASTERIO E ÁVILA, 2004).

A análise exploratória dos dados espaciais é realizada por meio do software GeoDa e foi realizada com o número de permutações aleatórias, conforme definido pela configuração padrão do software. Após a verificação inicial dos dados e análise de dependência e transbordamento espacial, é estimada a regressão econométrica da variável dependente contra algumas variáveis explicativas (descritas na seção 3.3).

\subsection{ABORDAGEM ECONOMÉTRICA}


A abordagem econométrica é desenvolvida por meio do software R. Como análise inicial roda-se a regressão por meio do método Mínimos Quadrados Ordinários (MQO) e verifica o comportamento de uma variável dependente contra algumas variáveis explicativas. Porém, esse método desconsidera a existência de dependência espacial entre as variáveis, o que pode tornar a estimativa viesada.

Existem algumas possibilidades de modelagem espacial para que 0 processo de transbordamento seja capturado adequadamente. Os modelos a serem testados são os de defasagem espacial, ou SAR (Spatial Auto Regressive), os modelos de erro autorregressivo espacial, ou SEM (Spatial Error Models), os modelos de alcance misto global, ou SAC (Spatial Lag Model with Spatial Auto Regressive Error) e o modelo espacial de Durbin, ou SDM (Spatial Durbin Model).

O primeiro deles, o modelo SAR, é expresso na sua versão mista da seguinte forma (ALMEIDA, 2004):

$$
y_{i}=\rho W y+X \beta+\varepsilon
$$

Em que $\rho$ é o coeficiente autorregressivo espacial que varia entre -1 e 1 , Wyé um vetor $n$ por 1 de defasagens espaciais para a variável dependente, e $\mathrm{X}$ é uma matriz de variáveis explicativas exógenas. Na equação 3 , o valor da variável dependente observado em uma determinada região $i$ é determinado pela média de valores da variável dependente na vizinhança, pelos valores das variáveis explicativas exógenas e ainda pelo termo de erro.

Modelos SEM possuem a seguinte especificação:

$$
y_{i}=X \beta+u
$$

Os resíduos dessa equação possuem uma estrutura autorregressiva na seguinte forma:

$$
u=\lambda W u+\varepsilon
$$

Onde $\lambda$ representa $o$ coeficiente escalar do erro espacial e indica a intensidade da autocorrelação espacial entre os resíduos da equação observada. Esse parâmetro mede o efeito médio dos erros dos municípios vizinhos em relação ao resíduo do município em questão. $\mathrm{E} \varepsilon$ representa o termo de erro normalmente distribuído com média zero e variância constante. A autocorrelação espacial nos modelos SEM aparece nos termos de erro (ALMEIDA, PEROBELLI E FERREIRA, 2008). 


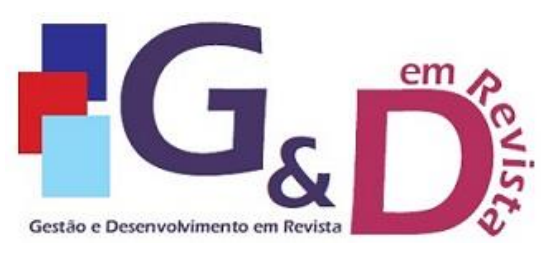

Gestão e Desenvolvimento em Revista V. 8, N. 2, jul-dez/2021, p. 90-108.

ISSN online: $2446-8738$

Artigo recebido em: 19/04/2021

Artigo aprovado em: 25/01/2022

O modelo SAC pode ser representado formalmente como (ALMEIDA, 2004):

$$
y_{i}=\rho W_{1} y+X \beta+u
$$

Neste modelo é incluída uma defasagem espacial e um termo de erro espacialmente dependente, o que faz com que um choque na região $j$ afete todas as regiões pelo processo de defasagem espacial e seja amplificado pelo erro espacial.

O modelo SDM tem a seguinte especificação (ALMEIDA, 2004):

$$
y_{i}=\rho W y+X \beta+W X \theta+\varepsilon
$$

Este modelo considera a existência de defasagem espacial do tipo WX, ou seja, incorpora o transbordamento espacial através da defasagem das variáveis independentes, supondo a possibilidade de omissão de variáveis relevantes que sejam correlacionadas com alguma das variáveis explicativas inclusas no modelo.

A matriz $W$ é a matriz ponderada de pesos espaciais. Quando o valor de $\lambda$ é igual a zero não existe autocorrelação espacial do erro, e quando $\lambda$ é diferente de zero o choque de um município se espalha para os municípios vizinhos (REY E MONTOURI, 1999).

Como testes diagnósticos são utilizados os critérios Akaike (AIC - Akaike Information Criterion) e Schwartz (BIC - Bayesian Information Criterion) para a escolha entre os modelos, cuja interpretação é de que quanto menores os valores, melhor.

\subsection{BASE E TRATAMENTO DE DADOS}

Nesse trabalho, a descrição das variáveis, seus sinais esperados e a fonte dos dados são apresentados no Quadro 1. Os sinais esperados das variáveis são baseados na literatura existente.

A variável que indica a quantidade de médicos para cada mil habitantes $(M E D)$, se encontra na dimensão Longevidade, visto que a disponibilidade de médicos é um indicador de acesso à saúde que pode afetar a expectativa de vida ao nascer. O percentual da população em domicílios com banheiro e água encanada (BAM) pertence à mesma dimensão, pois a questão do saneamento torna-se fundamental na medida em que pessoas sem acesso às condições básicas podem ter a sua saúde comprometida. 


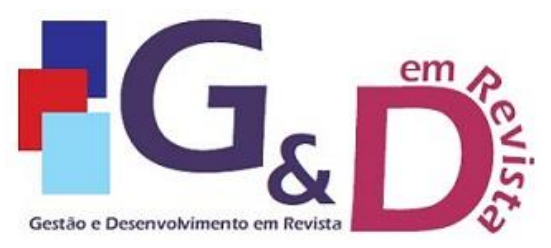

Gestão e Desenvolvimento em Revista V. 8, N. 2, jul-dez/2021, p. 90-108.

ISSN online: $2446-8738$

Artigo recebido em: 19/04/2021

Artigo aprovado em: 25/01/2022

Na dimensão Educação encontra-se o percentual de mulheres de 10 a 17 anos que tiveram filhos ( $F E R T)$, tendo em vista que a gravidez na adolescência se torna um agravante da baixa escolaridade entre jovens, uma vez que as mães podem deixar de estudar para cuidar do filho e/ou trabalhar. Além disso, a adolescente pode abandonar a escola por se sentir constrangida ou desmotivada caso não receba o apoio necessário. Nessa mesma dimensão foi considerada a taxa de atividade da população de 10 a 14 anos (PEA) como uma variável relacionada ao desenvolvimento, pois o trabalho infantil, apesar de contribuir para a renda familiar, pode gerar atraso e/ou abandono escolar por parte da criança.

Quadro 1 - Características das variáveis explicativas do IDHM

\begin{tabular}{|c|c|c|c|c|}
\hline Variável & Descrição & Proxy & $\begin{array}{c}\text { Sinal } \\
\text { Esperado }\end{array}$ & Fonte \\
\hline IDHM & $\begin{array}{c}\text { Índice de Desenvolvimento } \\
\text { Humano Municipal - IDHM (2010) }\end{array}$ & * & & ATLASBRASIL \\
\hline MEDi & $\begin{array}{l}\text { Número de médicos/1000 hab. } \\
\qquad(2010)\end{array}$ & I ngevidado & + & $\begin{array}{c}\text { DATASUS, } \\
\text { ATLASBRASIL }\end{array}$ \\
\hline $\mathrm{BAN}_{\mathrm{i}}$ & $\begin{array}{l}\text { Porcentagem da população em } \\
\text { domicílios com banheiro e água } \\
\text { encanada (2010) }\end{array}$ & & + & ATLASBRASIL \\
\hline $\mathrm{FERT}_{\mathrm{i}}$ & $\begin{array}{l}\text { Porcentagem de mulheres de } 10 \text { a } \\
17 \text { anos que tiveram filhos (2010) }\end{array}$ & \multirow{4}{*}{ Escolaridade } & - & ATLASBRASIL \\
\hline$P A_{i}$ & $\begin{array}{l}\text { Porcentagem PEA de } 10 \text { a } 14 \text { anos } \\
\text { com relação ao total da população } \\
\text { de } 10 \text { a } 14 \text { anos (2010) }\end{array}$ & & - & ATLASBRASIL \\
\hline $\mathrm{JOV}_{\mathrm{i}}$ & $\begin{array}{l}\text { Porcentagem população jovem - } 0 \\
\text { a } 09 \text { anos (2010) }\end{array}$ & & - & ATLASBRASIL \\
\hline$R_{\text {R }}$ & $\begin{array}{l}\text { Porcentagem dos ocupados no } \\
\text { setor agropecuário - } 18 \text { anos ou } \\
\text { mais (2010) }\end{array}$ & & - & ATLASBRASIL \\
\hline$I_{D A D E}$ & $\begin{array}{l}\text { Tempo entre a data de Instalação } \\
\text { do município e o ano de } 2010\end{array}$ & * & + & IBGE \\
\hline
\end{tabular}

Fonte: Elaborado pelos autores. 


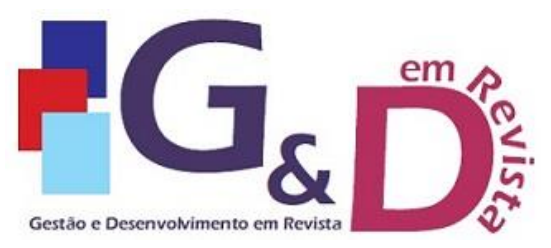

Gestão e Desenvolvimento em Revista V. 8, N. 2, jul-dez/2021, p. 90-108. ISSN online: $2446-8738$

$\mathrm{Na}$ dimensão da Renda, o percentual da população que possui entre 0 e 9 anos de idade (JOV) visa captar o possível efeito negativo que uma alta proporção de dependentes pode representar à ascensão econômica, haja vista que não fazem parte da força produtiva.

Tendo em vista as teorias que consideram que quanto mais desenvolvida uma região, menor a participação da agropecuária na geração de renda e maior a população urbana com relação à rural, a proporção da PEA ocupada no setor agropecuário $(R U R)$ pode ser um indicador de desenvolvimento. Considerando ainda que o desenvolvimento de uma região passa por diferentes etapas, a idade de um município (IDADE) pode representar o quanto ele já avançou nesse processo, especialmente porque municípios mais antigos tendem a ter maior infraestrutura para receber empreendimentos e ser sede de prestação de serviços públicos.

\section{RESULTADOS E DISCUSSÕES}

A amostra conta com dados de todos os 217 municípios do estado do Maranhão para o ano de 2010. Como variável dependente foi considerada neste estudo o IDHM, no qual os municípios que apresentaram o melhor e pior índice foram respectivamente, São Luiz e Fernando Falcão. A Tabela 1 apresenta algumas estatísticas descritivas dos dados utilizados.

Tabela 1 - Estatística descritiva dos dados

\begin{tabular}{cccccc}
\hline Variável & Média & $\begin{array}{l}\text { Desvio } \\
\text { Padrão }\end{array}$ & Máximo & Mínimo & Coeficiente de Variação \\
\hline IDHM & 0,572 & 0,046 & 0,768 & 0,443 & 0,081 \\
MED & 0,358 & 0,203 & 1,299 & 0,046 & 0,567 \\
BAN & 37,430 & 16,552 & 87,480 & 4,930 & 0,442 \\
FERT & 4,460 & 1,696 & 12,270 & 0,970 & 0,380 \\
PEA & 10,052 & 5,045 & 31,218 & 1,080 & 0,502 \\
JOV & 21,059 & 2,332 & 28,510 & 14,971 & 0,111 \\
RUR & 50,240 & 14,597 & 84,090 & 1,830 & 0,291 \\
IDADE & 48,000 & 24,707 & 71,000 & 13,000 & 0,515 \\
\hline
\end{tabular}

Fonte: Elaborado pelos autores. 
Não são verificados problemas de "ilhas", ou seja, municípios sem quaisquer outras divisas, o que, de acordo com Almeida (2004), poderia causar problema de perda de graus de liberdade. Na Tabela 2 apresenta-se a estatística I de Moran Global calculado com a matriz de pesos espaciais 'rainha'. A opção por essa matriz de pesos foi feita por ser a matriz que apresentou o maior I de Moran, indicando ser a melhor para analisar a autocorrelação espacial.

Tabela 2 - Indicador de autocorrelação espacial - I de Moran Global

\begin{tabular}{cccccc}
\hline Indicador & Coeficiente & Média & Desvio Padrão & Z-valor & P-Valor \\
\hline I de Moran L. & 0,2944 & $-0,0075$ & 0,0437 & 6,9087 & 0,0010 \\
\hline
\end{tabular}

Fonte: Elaborado pelos autores.

Os resultados indicam a existência de autocorrelação espacial positiva $(0,2944)$, deste modo podemos rejeitar a hipótese de distribuição aleatória espacial de IDHM para o estado do Maranhão no ano de 2010.

Figura 1 - Diagrama de dispersão de Moran para o IDHM de 2010 no estado do Maranhão

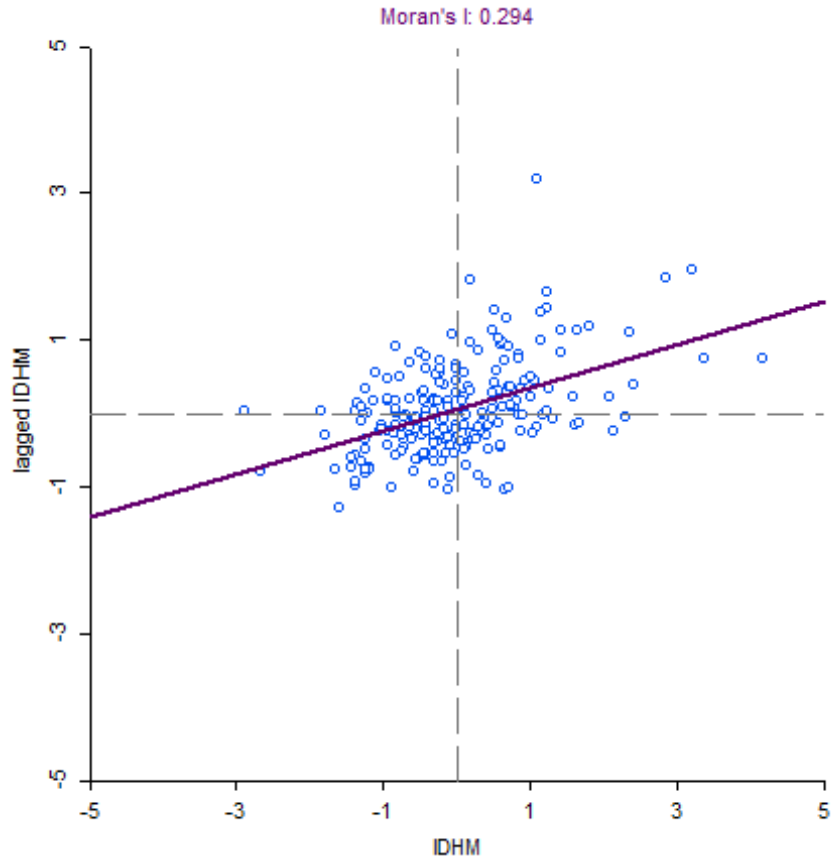

Fonte: Elaborado pelos autores.

No diagrama de dispersão de Moran (Figura 1) podem ser vistos agrupamentos (clusters) representados em quatro tipos de associações espaciais, 


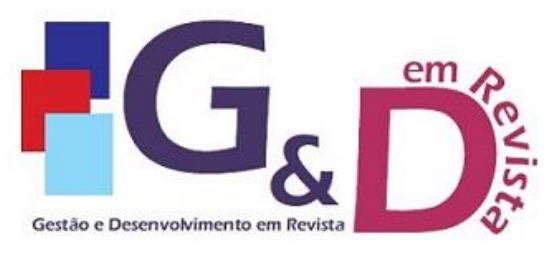

Gestão e Desenvolvimento em Revista V. 8, N. 2, jul-dez/2021, p. 90-108. ISSN online: $2446-8738$

sendo, Alto-Alto ( $A A$ - primeiro quadrante), Baixo-Baixo (BB - terceiro quadrante), Baixo-Alto ( $B A$ - segundo quadrante) e Alto-Baixo ( $A B$ - quarto quadrante).

$\mathrm{Na}$ Figura 2 observa-se a formação de clusters estatisticamente significativos, onde se destacam concentrações Alto-Alto na mesorregião Sul maranhense nas cidades de Alto Parnaíba e Tasso Fragoso. Na mesorregião Oeste apresentam-se dois clusters AA, um nas cidades de Porto Franco, Estreito, Lajeado Novo, São João do Paraíso e Campestre do Maranhão e outro cluster formado por Divinópolis, Imperatriz, Senador La Rocque, João Lisboa, Governador Edison Lobão, São Francisco do Brejão e Cidelândia. Na mesorregião Norte Maranhense encontram-se mais oito cidades, sete delas ficam na região metropolitana de São Luis: Raposa, Bacabeira, Rosário, Axixá, São José de Ribamar, Paço do Lumiar e São Luiz; e outra isolada, Central do Maranhão. E também isolado o município de Bernardo do Mearim localizado na mesorregião Centro Maranhense.

Figura 2 - Mapa de Cluster para o IDHM de 2010 do estado do Maranhão
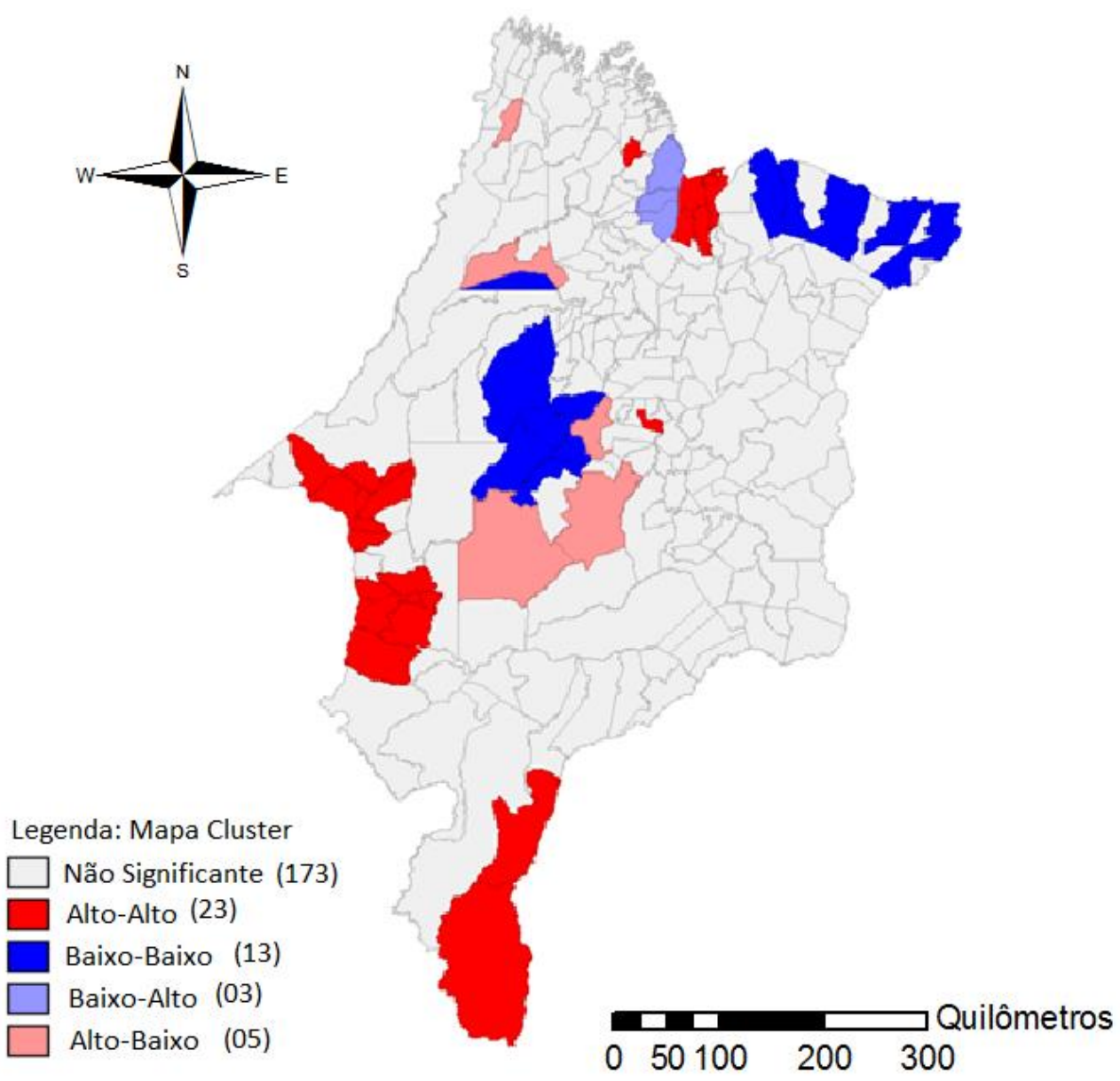

Fonte: Elaborado pelos autores. 
Já as formações de clusters Baixo-Baixo são verificadas na mesorregião Norte, nos municípios de Primeira Cruz, Humberto de Campos, Tutóia, Barreirinhas. $\mathrm{Na}$ mesorregião Leste: Araioses, São Bernardo. $\mathrm{Na}$ mesorregião Centro Maranhense: Marajá do Sena, Lagoa Grande do Maranhão, Arame, Itaipava do Grajaú, Santa Luzia, Paulo Ramos. E na mesorregião Oeste Maranhense, também isolada, o município de Governador Newton Bello.

A formação de clusters Alto-Alto nas regiões sul, oeste, norte, centro e metropolitana de São Luis pode ser explicada em parte pelo cultivo da soja no sul do estado e pela indústria desenvolvida da capital. Clusters Baixo-Baixo foram observados nas regiões norte, leste, centro e oeste do estado, possível reflexo das condições climáticas desfavoráveis e ocupações tardias.

Para melhor analisar esses dados, será utilizado o modelo econométrico de MQO, a fim de identificar se as variáveis explanatórias são estatisticamente adequadas para justificar as influências aqui apresentadas, sem considerar a correlação espacial.

Tabela 3 - Resultado da Regressão por MQO

\begin{tabular}{cccc}
\hline Variável & Coeficiente & Probabilidade & Desvio Padrão \\
\hline Constante & 0,7361 & 0,00 & 0,0311 \\
MED & $-0,0122$ & 0,20 & 0,0095 \\
BAN & 0,0006 & 0,00 & 0,0001 \\
FERT & $-0,0009$ & 0,39 & 0,0010 \\
PEA & 0,0004 & 0,30 & 0,0003 \\
JOV & $-0,0053$ & 0,00 & 0,0010 \\
RUR & $-0,0015$ & 0,00 & 0,0001 \\
IDADE & 0,0002 & 0,00 & 0,0000 \\
\hline
\end{tabular}

Fonte: Elaborado pelos autores. 
Tabela 4 - Diagnósticos Regressão MQO Teste de normalidade dos erros

\begin{tabular}{|c|c|c|c|}
\hline \multicolumn{2}{|c|}{ Estatística Jarque-Bera } & 2,8682 & 0,2383 \\
\hline \multicolumn{2}{|c|}{ Teste de heteroscedasticidade dos coeficientes } & Coeficiente & Probabilidade \\
\hline \multicolumn{2}{|c|}{ Teste Breusch-Pagan } & 17,957 & 0,012 \\
\hline \multicolumn{2}{|c|}{ Teste Koenker-Bassett } & 14,773 & 0,039 \\
\hline \multirow[t]{2}{*}{ Matriz de peso espacial $\mathrm{W}$} & Teste & & \\
\hline & LM (Lag) & 48,438 & 0,000 \\
\hline \multirow{4}{*}{$\begin{array}{l}\text { Matriz Rainha com uma } \\
\text { contiguidade }\end{array}$} & LM Robusto (Lag) & 1,691 & 0,193 \\
\hline & LM (Erro) & 84,008 & 0,000 \\
\hline & LM Robusto (Erro) & 37,261 & 0,000 \\
\hline & LM (SARMA) & 85,699 & 0,000 \\
\hline
\end{tabular}

Fonte: Elaborado pelos autores.

No diagnóstico da regressão (Tabela 4) verifica-se que o teste de JarqueBera apresenta normalidade dos erros, e os testes Breusch-Pagan e KoenkerBassett indicam que os resíduos da regressão são homocedásticos. Ainda cabe citar que a condição de multicolinearidade $(59,9077)$ apresentou-se aceitável.

Com o teste do I de Moran para a aleatoriedade espacial dos resíduos podese verificar que o modelo de regressão tradicional é viesado para tratar a dependência espacial. De acordo com os resultados apresentados na Tabela 5, há evidências da existência de autocorrelação espacial nos resíduos.

Tabela 5 - Teste de Autocorrelação Espacial dos Resíduos

\begin{tabular}{ccc}
\hline Indicador & Valor da estatística & $\boldsymbol{p}$-valor \\
\hline I de Moran & 0,396 & 0,002 \\
\hline
\end{tabular}

Fonte: Elaborado pelos autores.

Considerar a questão espacial é importante para explicar a relação entre as variáveis, pois o modelo de regressão tradicional mostrou-se inadequado. Dessa forma, parte-se para a estimação de modelos que levem em conta essa dependência. Serão considerados os modelos espaciais de alcance global (SAC, SAR e SEM) e a escolha do modelo adequado será feita por meio da comparação dos resultados dos critérios de informação Akaike (AIC) e Schwartz (BIC), apresentados na Tabela 6 . 


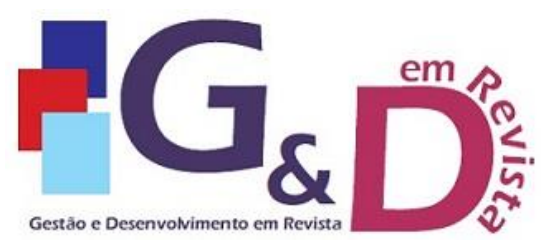

Gestão e Desenvolvimento em Revista V. 8, N. 2, jul-dez/2021, p. 90-108. ISSN online: $2446-8738$ Artigo recebido em: 19/04/2021 Artigo aprovado em: 25/01/2022

Tabela 6 - Critérios de Informação

\begin{tabular}{ccccc}
\hline Critério & SAC & SAR & SEM & SDM \\
\hline AIC & $-1.043,0748$ & $-1.015,5843$ & $-1.044,6917$ & $-1.037,2959$ \\
BIC & $-1.005,8959$ & $-981,7853$ & $-1.010,8927$ & $-979,8377$ \\
\hline
\end{tabular}

Fonte: Elaborado pelos autores.

Conforme sugerido nos testes para dependência espacial, o modelo de erro espacial (SEM) mostrou-se o mais adequado, pois quanto menor o valor dos critérios de informação, melhor o modelo. Os resultados da regressão de tal modelo estão apresentados na Tabela 7.

As variáveis MED e FERT não foram significativas ao nível de $5 \%$. Por outro lado, todas as outras variáveis mostraram-se significativas para o modelo. Todas as variáveis estatisticamente significativas para 0 modelo apresentaram 0 sinal esperado, com exceção da variável PEA que apresentou sinal positivo, o que pode sugerir que, no estado do Maranhão, os jovens de 10 a 14 anos ainda são uma parte considerável da formação de renda nos domicílios.

Tabela 7 - Modelo de Erro Espacial

\begin{tabular}{cccc}
\hline Variável & Coeficiente & Probabilidade & Desvio Padrão \\
\hline Constante & 0,7022 & 0,00 & 0,0295 \\
MED & $-0,0003$ & 0,97 & 0,0075 \\
BAN & 0,0006 & 0,00 & 0,0001 \\
FERT & $-0,0006$ & 0,45 & 0,0008 \\
PEA & 0,0006 & 0,03 & 0,0002 \\
JOV & $-0,0041$ & 0,00 & 0,0010 \\
RUR & $-0,0016$ & 0,00 & 0,0001 \\
IDADE & 0,0002 & 0,00 & 0,0000 \\
Lambda & 0,6456 & 0,00 & 0,0636 \\
\hline
\end{tabular}

Fonte: Elaborado pelos autores.

A variável $B A N$ apresentou sinal positivo, o que indica que o IDHM aumenta nos municípios onde houver elevação na proporção do número de domicílios com banheiros e água encanada. No sentido contrário, os coeficientes para JOV e RUR apresentaram sinais negativos, o que sugere tanto a maior proporção de jovens de 0 a 9 anos em relação à população total do município, como a maior proporção dos ocupados no setor agropecuário (18 anos ou mais), contribuem para um baixo 
IDHM. Também é verificado que a variável IDADE influencia positivamente os municípios, o que sugere que quanto mais antigo o município, maior o seu IDHM.

Portanto, os resultados mostraram que o percentual da população até 9 anos de idade e a PEA da agropecuária relacionam-se negativamente com o IDHM dos municípios maranhenses. Já a proporção de domicílios com banheiro e a idade do município impactam positivamente o desenvolvimento.

O parâmetro $\lambda$ apresentou-se significativo, indicando que a ocorrência de um choque em um município causará um transbordo para outros municípios. Ou seja, verificou-se dependência espacial das variáveis, indicando que elas afetam também os municípios vizinhos.

\section{CONSIDERAÇÕES FINAIS}

No presente trabalho buscou-se analisar a dependência espacial do desenvolvimento econômico em termos de IDHM nos municípios do estado do Maranhão, no ano de 2010, e as variáveis que podem tê-lo afetado.

Os resultados corroboraram as teorias que consideram o desenvolvimento econômico multidimensional, confirmando que os determinantes de qualidade de vida vão além da renda. Ademais, verificou-se haver dependência espacial, confirmando que a localização geográfica do município e a condição de desenvolvimento de seus vizinhos pode afetar o bem estar de sua população, conforme preconizado nas teorias de desenvolvimento regional.

O estudo também confirmou os achados de Lins et al. (2014) e Santos (2018) sobre dependência espacial na qualidade de vida maranhense. Longe de esgotar as pesquisas na área, percebe-se que o estado do Maranhão necessita uma política de distribuição das riquezas pelo estado, uma vez que possui regiões desenvolvidas, mas o estado como um todo não acompanhou a trajetória de desenvolvimento nacional em vinte anos.

\section{REFERÊNCIAS}

ALMEIDA, E.S. Curso de econometria espacial aplicada, ESALQ-USP, Piracicaba, 2004. 


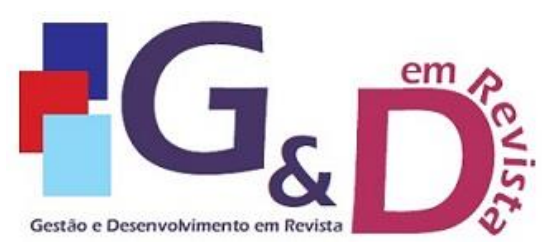

Gestão e Desenvolvimento em Revista V. 8, N. 2, jul-dez/2021, p. 90-108. ISSN online: $2446-8738$

ALMEIDA, E.S.; PEROBELLI, F.S.; FERREIRA, P.G.C. Existe Convergência Espacial da Produtividade Agrícola no Brasil? RER, Rio de Janeiro, v.46, n.1, p.3152, jan.-mar./2008.

ANSELIN, L. Local Indicators of Spatial Association - LISA. Geographical Analysis. Vol. 27, n. 2, pp. 93-115, abr./1995.

ATLAS BRASIL. Atlas do Desenvolvimento Humano no Brasil. Disponível em: http://www.atlasbrasil.org.br/. Acesso em: mar./2020.

ATLAS BRASIL. O IDHM. Disponível em:

http://www.atlasbrasil.org.br/acervo/atlas. Acesso em: mar./2020.

CAVALCANTE, L.R.; Produção teórica em economia regional: uma proposta de sistematização. Revista Brasileira de Estudos Regionais e Urbanos, v. 02, p. 932, 2008.

CLIFF, A.D.; ORD, J.K. Spatial processes: models and applications. Pion, London. 1981.

DATASUS. Indicadores e Dados Básicos. Disponível em:

http://tabnet.datasus.gov.br/cgi/idb2012/matriz.htm\#cober. Acesso em: mar./2020.

LINS, J.G.M.G.; LOURES, A.R.; LOMBARDI FILHO, S.C.; SILVA, M.V.B. Análise espacial da evolução do Índice de Desenvolvimento Humano nos municípios da região Nordeste. X Encontro de Economia Baiana - Economia Regional. Anais... Set. 2014.

LOBO, B. J. M. Análise espacial do desenvolvimento humano educacional dos municípios do Estado do Ceará nos anos 2000 e 2010. 2017. 49f. - Dissertação (Mestrado) - Universidade Federal do Ceará, Programa de Economia Profissional, Fortaleza (CE), 2017.

MONASTERIO, L.M.; ÁVILA, R.P. Uma Análise Espacial do Crescimento do Rio Grande do Sul (1939-2001). Revista Economia, Brasília - DF, v.5, n.2, p.269-296, jul.-dez./2004.

REY, S.J.; MONTOURY, B.D.U.S. Regional Income Convergence: A Spatial Econometric Perspective. Regional Studies, v. 33, n. 2, p. 143-156, Apr 1999.

PNUD BRASIL. O que é Desenvolvimento Humano? Disponível em: https://www.br.undp.org/content/brazil/pt/home/idh0/conceitos/o-que-edesenvolvimento-humano.html . Acesso em: jan./2021.

SANTOS, V.B. Análise espacial dos óbitos por tuberculose no estado do Maranhão. Dissertação de mestrado - Programa de Pós-graduação em Saúde Coletiva. UFMA. São Luís, 56 p., 2018. 

V. 8, N. 2, jul-dez/2021, p. 90-108. ISSN online: $2446-8738$

Artigo recebido em: 19/04/2021

Artigo aprovado em: 25/01/2022

SEN, A. Desenvolvimento como liberdade. São Paulo: Companhia das Letras, 2000.

SOUZA, N.J. Desenvolvimento Econômico. 5aㅡ Ed. São Paulo: Atlas, 2007.

1 